\title{
O-splines para analizar señales de oscilaciones de potencia
}

\author{
José Antonio De la O Serna \\ Universidad Autónoma de Nuevo León \\ Facultad de Ingeniería Mecánica y Eléctrica \\ jose.delaosr.uanl.edu.mx
}

\section{RESUMEN}

Se presenta una nueva familia de splines y sus derivadas, las cuales provienen de los diferenciadores pasabajas de la Transformada Discreta Taylor-Fourier (DTFT). Se llaman $O$-splines porque sus segmentos están espaciados en tramos de un ciclo de la frecuencia fundamental. Con ellas se analizan señales oscilatorias de potencia. Para ilustrar su aplicación y su progresiva exactitud se aplican para estimar fasores de voltajes, y para separar modos electromecánicos de oscilación en un sistema de potencia real. Con ellas se disminuye la complejidad computacional de la DTFT, ya que se aplica solamente un subconjunto de filtros. Los parámetros estimados ofrecen información dinámica mucho más rica que los métodos tradicionales. En particular, brindan una representación de estados para cada componente oscilatoria, y detección de eventos modulados en frecuencia. $\mathrm{Su}$ rendimiento en estimación se evalúa con un nuevo error llamado Error Fasorial Total (Total Phasor Error). Se concluye que esta técnica multiresolución ofrece una serie de soluciones de gradual exactitud para la estimación fasorial y la separación de modos de oscilación. Este nuevo marco matemático fusiona el área de medición fasorial con la de análisis de modos de oscilación en sistemas eléctricos de potencia que tradicionalmente han estado separadas en ingeniería eléctrica.

\section{PALABRAS CLAVE}

Transformada Discreta Taylor Fourier, fasor dinámico, modos electromecánicos, detección de envolvente compleja, bancos de filtros, análisis multiresolución, armónicas oscilatorias, $O$-splines, espacio de fase, oscilaciones de potencia, estimación de sincrofasores, separación tiempo-frecuencia, error fasorial total.

\section{ABSTRACT}

A new family of splines and their derivatives is presented, which come from the low-pass differentiators of the Discrete Taylor-Fourier Transform (DTFT). They are called $O$-splines because their segments are spaced in steps of a cycle from the fundamental frequency. With them oscillatory power signals are analyzed. To illustrate their application and their progressive accuracy, they are applied to estimate voltage phasors, and to separate electromechanical modes of oscillation in a real power system. With them, the computational complexity of the DTFT is reduced, since only a subset of filters is applied. The estimated parameters offer much richer dynamic information than traditional methods. In particular, they provide a representation of states for 
each oscillatory component, and detection of frequency modulated events. Its estimation performance is evaluated with a new error called Total Phasor Error. It is concluded that this multiresolution technique offers a series of solutions of gradual accuracy for the phasor estimation and the separation of oscillation modes. This new mathematical framework merges the area of phasor measurement with that of analysis of modes of oscillation in electrical power systems that have traditionally been separated in electrical engineering.

\section{KEYWORDS}

Discrete Taylor Fourier Transform, dynamic phasor, electromechanical modes, complex envelope detection, filter banks, multiresolution analysis, oscillatory harmonics, O-splines, phase space, power oscillations, synchrophasor estimation, time-frequency separation, total phasor error.

\section{INTRODUCCIÓN}

La medición de señales oscilatorias de sistemas eléctricos de potencia es considerada en la literatura como dos problemas independientes. Por una parte, hay muchos estudios para implementar mejores algoritmos para medir sincrofasores de señales de voltaje y de corriente bajo oscilaciones para las unidades de medición fasorial (PMUs) instaladas en las redes inteligentes de área amplia. Por la otra, existe una abundante literatura dedicada a mejorar el análisis de modos electromecánicos de oscilaciones de potencia. Este artículo propone los $O$-splines de la Transformada Discreta Taylor Fourier para resolver estos dos importantes problemas.

Ambos problemas tienen en común componentes de señal con energía espectral densa alrededor de ciertas frecuencias: de las frecuencias armónicas en el primer caso, y junto a las frecuencias de los modos de oscilación en el segundo. El problema básico de la transformada de Fourier (DFT) al tratar con componentes espectrales densas es que distorsiona tanto la amplitud como la fase. ${ }^{1}$ La DFT trabaja con exactitud solo cuando las señales tienen componentes con amplitud, fase y frecuencia constantes. La transformada Taylor Fourier (DTFT) resuelve esta limitación sustituyendo cada coeficiente de Fourier constante por un polinomio de Taylor de orden K durante la duración temporal de la ventana de observación. Esto le permite capturar la envolvente compleja de cada componente.

Subespacios más amplios se generan incluyendo términos de Taylor de más alto orden en cada componente armónica de la base de Fourier. ${ }^{2}$ Dado que los polinomios de Taylor de más alto orden incluyen los de orden inferior, cada término de Taylor incluido en el modelo de señal hace que el subespacio crezca gradualmente desde el anterior. Por tanto, las extensiones de polinomios de Taylor generan una escalera de espacios con elementos que se aproximan cada vez más a la señal pasabanda ideal. Inician con el más burdo, definido por los coeficientes complejos de la DFT, en el cual residen las señales periódicas. Al agregar más y más términos de Taylor al modelo de señal, se generan subespacios más vastos, conteniendo los anteriores. Entonces, los subespacios DTFT ofrecen mejores aproximaciones a las señales oscilatorias, y por tanto brindan parámetros dinámicos con errores cada vez más pequeños. Consecuentemente, los estimados obtenidos con polinomios de grados superiores son siempre más precisos y exactos en señales oscilatorias que los obtenidos con grados inferiores. 
En este trabajo (cuya versión en inglés se encuentra en ${ }^{3}$ ) se comparan los parámetros estimados con polinomios de Taylor de primer y tercer orden. Además, se demuestra que las respuestas impulsionales de los filtros DTFT son modulaciones a las frecuencias armónicas de las $O$-splines. Por tanto, nos concentramos en los diferenciadores pasabajas. Los $O$-splines son funciones definidas por segmentos polinomiales. En nuestro caso, cada ciclo es definido por un polinomio distinto.

En el lado espectral, la extensión de Taylor ensancha y aplana el lóbulo principal de los filtros DTFT. Como resultado de esta relajación espectral, los filtros de la DTFT son más adecuados para medir armónicas oscilatorias, ya que no las distorsionan ni en amplitud ni en fase. En cuanto a la descomposición de la señal, la DTFT está dotada de un banco de filtros con ganancias de diferenciador ideal en su banda de paso, y con bandas de paro con ganancia nula en el resto de las armónicas. Por tanto, en cada frecuencia armónica, los filtros actúan como diferenciadores ideales, con rechazo perfecto del resto de las armónicas.

Con respecto a la aplicación de estimación de sincrofasores, vale la pena mencionar que el estándar de sincrofasores ${ }^{4}$ fue escrito bajo la fuerte influencia de estimador tradicional DFT de un ciclo. ${ }^{5}$ Ahora el énfasis del estándar está puesto en el efecto del ruido en las estimaciones. ${ }^{6}$ Pero se defiende persistentemente el filtro de Fourier a pesar de sus conocidas limitaciones en condiciones oscilatorias (la infiltración de la imagen de la frecuencia fundamental, y la pérdida debida a su ganancia en forma de concha). ${ }^{7,8}$ Por estas razones, después de reconocer la buena regulación en estado estable del estándar de sincrofasores del IEEE, el Consejo de Coordinación de Electricidad del Oeste (Western Electricity Coordinating Council (WECC)) ${ }^{9}$ propuso mejorar los requisitos de rendimiento dinámico de los filtros usados en PMUs, a fin de garantizar mejores respuestas ante condiciones dinámicas, tales como vaivenes de potencia o armónicas cambiantes. Y propuso un filtro complejo de pasabanda adaptiva derivado de la teoría de bancos de filtros modulados exponencialmente, con respuestas impulsionales de 4 y 7 ciclos, y variaciones frecuenciales en el rango entre 40 a $80 \mathrm{~Hz}$. Aquí se propone la serie de respuestas impulsionales de los diferenciadores pasabanda DTFT para observar la dinámica de señales oscilatorias. Las soluciones ofrecidas pueden graduarse para mejorar la precisión y exactitud de los estimados, empezando con el filtro más burdo (DFT), y terminando con mucho mejores representaciones óptimas para las aplicaciones de protecciones (clase P) y de mediciones (clase M).

Por otra parte, la identificación de modos electromecánicos en oscilaciones de potencia ha sido estudiada por muchos investigadores a través de las siguientes aproximaciones: ${ }^{10}$ análisis lineal de oscilaciones atenuadas ringdown, ${ }^{11,12}$ análisis de modos, ${ }^{13} \mathrm{y}$ análisis no lineal. ${ }^{14,15}$ Estos métodos (Prony, Yule Walker, and Hilbert Wang) consideran los modos de oscilación como componentes de banda ancha localizados en cualquier parte del espectro, mientras que la DTFT ${ }^{16}$ particiona la frecuencia en un conjunto de intervalos disjuntos desde los cuales la envolvente compleja de cada modo de oscilación es observada a través de una descomposición de Taylor. Ofrece separación tiempo-frecuencia en modos de frecuencia fluctuante, y una parametrización en 
espacio de fase para las dinámicas de cada modo. Por supuesto, la frecuencia central de los filtros DTFT debe sintonizarse con la frecuencia media central de cada modo. ${ }^{17}$ El ejemplo analizado en la sección de resultados ilustra la nueva parametrización ofrecida por el análisis DTFT.

En este trabajo nos proponemos desarrollar un marco teórico que mejore la estimación de sincrofasores en corrientes y voltajes, así como la la separación de modos de oscilación en oscilaciones de potencia, motivados por la hipótesis de multiresolución, según la cuál a los $O$-splines de más alto grado les corresponde un error de aproximación mucho más pequeño, y en consecuencia estimaciones paramétricas más completas y de mejor calidad.

En lo que sigue, el modelo de señal en subespacios Taylor Fourier se presenta en la sección de metodología, con las respuestas impulsionales de los filtros, y sus respuestas en frecuencia. Esto se hace para los diferenciadores pasabanda de primer y tercer orden. En la sección de resultados se muestran los fasores dinámicos de la DTFT estimados punto a punto con polinomios de Taylor de primer y tercer orden y obtenidos de las señales de voltaje durante una oscilación. Las estimaciones de amplitud, fase y frecuencia se ilustran para mostrar cómo mejora la exactitud de las estimaciones. Finalmente, en la sección de resultados, la DTFT es usada para separar los modos electromecánicos de la oscilación de potencia. El análisis ilustra los estimados de amplitud y fase de los primeros dos modos, junto con sus primeras derivadas. Concluye que ambas aplicaciones de los $O$-splines enriquecen la representación paramétrica de estos eventos dinámicamente complejos.

\section{METODOLOGÍA UTILIZADA}

Se utiliza el método conocido como multiresolución, que consiste en definir una escalera de subsepacios ${ }^{18}$ para aproximar con más y más precisión las señales analizadas. En nuestro caso los subespacios en cada frecuencia armónica son graduados simplemente por el orden del polinomio de Taylor utilizado en la DTFT. En esta sección se desarrolla esta idea.

La expansión del fasor estático al dinámico y sus derivadas se presentó originalmente en ${ }^{19}$. Esto condujo a la extensión del subespacio de Fourier a los de Taylor Fourier presentados en ${ }^{20}$. Esta puede ser aquí brevemente introducida iniciando desde el tradicional modelo de señal de Fourier de orden cero:

$$
x_{0}=\Phi_{0} \xi_{0}
$$

donde $\Phi_{0}$ es la matriz de Fourier $W_{N}$ de $N \times N$ elementos, y $\xi_{0}$ contiene los coeficientes de Fourier de $x_{0}$.

Los elementos de $W_{N}$ son $w_{n k}=e^{j \frac{2 \pi}{N} k n}$, para $n, k=0,1, \ldots, N-1$, donde $N$ es el número de muestras por ciclo fundamental.

Se extiende el subespacio anterior incluyendo el primer término de Taylor. El modelo de señal para esta primera extensión es entonces como muestra la ecuación 2, en donde las matrices diagonales $I$ y $T$ contienen las muestras de los términos de Taylor de cero y primer orden, respectivamente, tomados en un intervalo centrado de dos ciclos. Y el subvector $\dot{\xi}_{N} \mathrm{~N}$ contiene las derivadas de los primeros coeficientes Taylor Fourier en $\xi_{N}$. 


$$
\begin{aligned}
& x_{1}=\Phi_{1} \xi_{1} \\
& =\left(I\left(\begin{array}{c}
W_{N} \\
W_{N}
\end{array}\right) \quad T\left(\begin{array}{l}
W_{N} \\
W_{N}
\end{array}\right)\right)\left(\begin{array}{l}
\xi_{N} \\
\dot{\xi}_{N}
\end{array}\right)
\end{aligned}
$$

Las siguientes extensiones se obtienen incluyendo más y más términos de Taylor. El modelo de señal para la $K$-ésima extensión es como sigue:

$$
\begin{aligned}
x_{K} & =\Phi_{K} \xi_{K} \\
& \left.=\left(\begin{array}{c}
W_{N} \\
W_{N} \\
\vdots \\
W_{N}
\end{array}\right) \quad T\left(\begin{array}{c}
W_{N} \\
W_{N} \\
\vdots \\
W_{N}
\end{array}\right) \quad \cdots \quad \frac{1}{K !} T^{K}\left(\begin{array}{c}
W_{N} \\
W_{N} \\
\vdots \\
W_{N}
\end{array}\right)\right)\left(\begin{array}{c}
\xi_{N} \\
\dot{\xi}_{N} \\
\vdots \\
(K) \\
\xi_{N}
\end{array}\right)
\end{aligned}
$$

donde la matriz $\Phi_{K}$ de $(K+1) N \times(K+1) N$ contiene los vectores de la base del subespacio extendido, el subvector $\xi N$ contiene los coeficientes Taylor Fourier de K ésimo grado, y los subvectores $\xi(\mathrm{k}) N, k=1, \ldots K$ sus progresivas derivadas. Note que la extensión conlleva una prolongación cíclica del modelo de señal, de $C=1$ a $C=K+1$ ciclos.

Debido a la oblicuidad introducida por los términos de Taylor, las bases extendidas ya no son ortogonales, y por tanto, para $K \geq 1$, se requiere un par de bases biortogonales para la proyección ortogonal: ${ }^{21}$ una base para la antitransformada en (3) y su dual para la transformada:

$$
\widehat{\xi}=\widetilde{\Phi}^{H} x
$$

donde $\widetilde{\Phi}$ es la matriz dual de $\Phi$ dada por:

$$
\widetilde{\Phi}=\Phi\left(\Phi^{H} \Phi\right)^{-1}
$$

tal que $\tilde{\Phi}^{H} \Phi=I$.

En ${ }^{22}$ la matriz $\Phi_{K}$ en (3) es factorizada como sigue:

$$
\begin{aligned}
\Phi_{K} & =\Upsilon_{K} \Omega_{K} \\
& =\left(\begin{array}{cccc}
I & T_{1} & \ldots & { }_{K !}^{1} T_{1}^{K} \\
I & T_{2} & \ldots & \frac{1}{K !} T_{2}^{K} \\
\vdots & \vdots & \ddots & \vdots \\
I & T_{C} & \ldots & \frac{1}{K !} T_{C}^{K}
\end{array}\right)\left(\begin{array}{cccc}
W_{N} & 0 & \ldots & 0 \\
0 & W_{N} & \ldots & 0 \\
\vdots & \vdots & \ddots & \vdots \\
0 & 0 & \ldots & W_{N}
\end{array}\right)
\end{aligned}
$$

en la cual las submatrices cuadradas $T_{i}, i=1,2, \ldots, \mathrm{C}$ son las submatrices diagonales descendientes de $N \times N$ elementos, extraídas de la matriz diagonal $T$ en (3). Note que $\frac{1}{k !} T_{i}^{k}$ contiene la $i$-ésima pieza cíclica del $k$-ésimo término de Taylor. En esta factorización, el operador Taylor Fourier $\Phi_{K}$ es claramente separado en dos matrices: $\Omega_{K}$ del operador de Fourier y $\Upsilon_{K}$ del de Taylor.

Esta factorización fue propuesta en ${ }^{22}$ para reducir los cálculos de $\widetilde{\Phi}$, ya que para la forma factorizada se tiene:

$$
\widetilde{\Phi}=\Upsilon\left(\Upsilon^{H} \Upsilon\right)^{-1} \frac{\Omega}{N}=\widetilde{\Upsilon} \frac{\Omega}{N}
$$


En este artículo, se propone la formulación de la DTFT en (7) como un método de diseño de filtros pasabajas (diferenciadores pasabajas máximamente lisos) en $\widetilde{\Upsilon}$. Note que $\widetilde{\Phi}$ preserva la estructura de (6), puesto que los vectores de $\widetilde{\Upsilon}$ son combinaciones lineales de los vectores en $\Upsilon$. En consecuencia, los vectores en $\widetilde{\Phi}$ son simples modulaciones armónicas de las subdiagonales verticales de $\widetilde{\Upsilon}$. En otras palabras, esta factorización demuestra que el subconjunto de vectores banda base en $\tilde{\Upsilon}$ constituyen las envolventes de los correspondientes subconjuntos de vectores en las frecuencias armónicas de la DTFT.

Esta contribución es importante para aplicaciones donde la energía está presente en un pequeño número de frecuencias. Este es el caso de señales de sistemas de potencia, ya que la aplicación de sincrofasores solamente requiere los filtros pasabanda en la frecuencia fundamental, ya que tienen buen rechazo en las frecuencias armónicas; y la de oscilaciones de potencia solo requiere filtros en cada modo. En estas aplicaciones, la DTFT se implementa con una gran reducción de complejidad computacional, ya que se reduce a la aplicación de un pequeño numero de filtros de respuesta impulsional finita (FIR). Si se requiere estimar hasta la segunda derivada, se requieren 3 filtros FIR de longitud $(K+1) N$ por frecuencia observada.

Con respecto a la sensibilidad del ruido de los filtros DTFT, se puede afirmar $16,19,20$ que para señales con tasa de señal a ruido (SNR) arriba de $60 \mathrm{~dB}$, el error medio cuadrático de los parámetros estimados es despreciable.

\section{Error de aproximación}

La mejor señal aproximada a través de la descomposición DTFT se obtiene a través de la matriz de proyección $P=\Phi \widetilde{\Phi}^{H}$, , pues se tiene:

$$
\widehat{x}=\Phi \widehat{\xi}=\Phi \widetilde{\Phi}^{H} x
$$

El teorema de Pitágoras se cumple para el error de aproximación $e=x-\widehat{x}$, con

$$
\|\in\|^{2}=\|x\|^{2}-\hat{\xi}^{H} \Phi^{H} \Phi \widehat{\xi}
$$

y normalizando con respecto a la energía de la señal,

$$
\|\epsilon\|^{2}=1-\frac{\hat{\xi}^{\prime \prime} \Phi^{\prime \prime} \Phi \widehat{\xi}}{\|x\|^{2}}
$$

El error cuadrático medio anterior considera el error de la proyección sobre el subespacio total considerado, es decir el subespacio generado por todos los vectores de la base en $\Phi$.

Es también posible medir el error de aproximación de la proyección en el subespacio generado solamente por los vectores de la base asociados con la frecuencia fundamental, que generan el subespacio fundamental. Esto es posible tomando la submatriz $\Phi_{1}$ que contiene tales vectores, y los correspondientes estimados $\xi_{1}$. Esta medida será llamada Error Fasorial Total (TPE, por sus siglas en Inglés), ya que mide la energía faltante a la señal aproximada del subespacio fundamental. Esto, a su vez, corresponde a la Distorsión Total (TD) asociada a la componente fundamental. 
Entonces se tiene:

$$
\left\|\epsilon_{1}\right\|=\sqrt{1-\frac{\hat{\xi}_{1}^{H} \Phi_{1}^{H} \Phi_{1} \hat{\xi_{1}}}{\|x\|^{2}}}
$$

Para una señal dada $x$, el mejor estimador fasorial es entonces el que minimice el TPE. Como puede verse, la definición del TPE es la más general de las distorsiones, pues toma en cuenta cualquier tipo de energía espectral presente en la señal: subarmónicas, interarmónicas, y cualquier tipo de ruido. Para señales periódicas, el TPE se reduce a la distorsión armónica total (THD), ${ }^{23}$ y para sinusoidales de estado estable el TPE se reduce al error vectorial total (TVE) del estándar de sincrofasores. ${ }^{4}$ Para las señales de prueba del estándar, el TPE es la mejor medida de error, y no se correlaciona con el TVE. Una de las fuertes limitaciones del TVE es que solo se puede obtener cuando el fasor dinámico de la señal de prueba es conocido. El TPE se calcula con los datos de la señal dada y la aproximada, y puede usarse para comparar diferentes algoritmos, sin que el fasor dinámico subyacente de la señal sea conocido, como en el caso del TVE. Además, el TPE tiene otras dos ventajas: depende del conjunto total de parámetros

estimados $\widehat{\xi}$, y puede calcularse para intervalos de duración más cortos que el intervalo usado en la estimación.

\section{Respuesta impulsional y de frecuencia de los filtros FIR}

Cuando $\mathrm{K}$ aumenta, los modelos de señal DTFT ofrecen una serie de subespacios de aproximación V K, que forman una escalera de espacios, ${ }^{18}$ con un orden anidado: $V_{0} \subset V_{1} \subset V_{2} \cdots \subset V_{K}$, iniciando con el subespacio central $V_{0}$ de la DFT, y progresando gradualmente como $V_{K}=V_{K-1} \oplus W_{K-1}$, donde $\mathrm{W} \mathrm{K}_{-1}$ es el complemento ortogonal de $V_{K-1}$ en $V_{K}$. Entonces, iniciando con la aproximación más burda, ellos ofrecen gradualmente aproximaciones de más alta resolución. La principal diferencia entre la DTFT y el análisis multiresolución de onduletas, es que los $O$-splines separan el espectro en bandas uniformes, centradas en las frecuencias armónicas, mientras que las onduletas las separan en bandas diádicas.

Como las respuestas impulsionales de los filtros DTFT en cada frecuencia armónica son simples versiones moduladas de las de la banda base, concentramos nuestra atención en esta banda, para luego entender la operación de los filtros pasabanda.

La gráfica superior de la figura 1 ilustra los $O$-splines para diferentes grados de Taylor $K=0,1, \ldots, 11$. Éstas fueron calculadas con $\mathrm{N}=20$ muestras por ciclo. Puede demostrarse de ${ }^{7}$ que forman una nueva familia de splines con $C=K+1$ ciclos. Las primeras dos corresponden a los splines $\mathrm{B}$ (B-splines) de cero y primer orden, ${ }^{24}$ pero luego difieren de éstas cuando $K \geq 2$, ya que las $\mathrm{O}$ convergen al Seno Cardenal (Cardinal Sine), y no a la Gausiana como las B, como puede corroborarse con la curva con $K=11$. Inician con la ventana rectangular del filtro Fourier de un ciclo, luego la triangular de dos ciclos, como el ejemplo de filtro clase P del estándar de sincrofasores, ${ }^{4} \mathrm{y}$ entonces sus formas evolucionan hasta la función Seno Cardenal, cuando $K \rightarrow \infty$. De hecho, el quinto filtro de 6 ciclos es muy similar al filtro clase $\mathrm{M}$ del estándar ${ }^{4}$. 


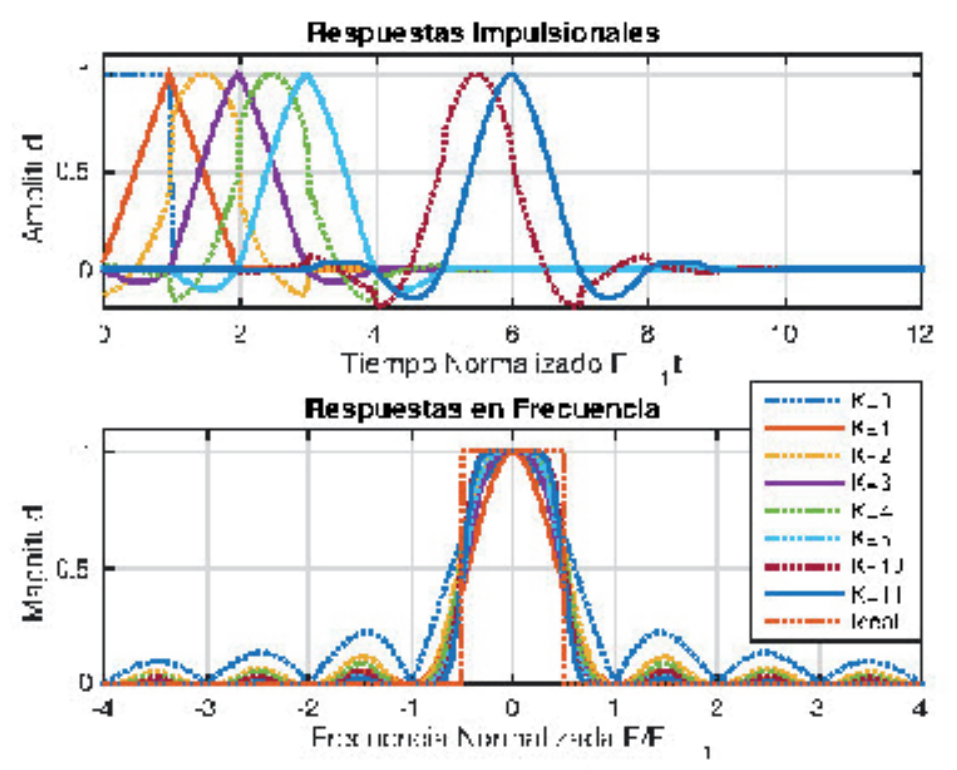

Fig. 1. O-splines (gráfica superior) y respuestas frecuenciales (gráfica inferior) de los filtros DTFT pasabajas, $K=0,1, \ldots, 5$, y 11 .

Por otro lado, la gráfica inferior de la figura 1 ilustra las respuestas en frecuencia de los $O$-splines. Como se puede ver, la secuencia inicia con la función Seno Cardenal, correspondiente a la ventana rectangular, seguida por su cuadrado que corresponde a la ventana triangular. Después de éstas las respuestas en frecuencia empiezan a tener pasabanda y bandas de paro cada vez más planas. Esta propiedad es muy importante para el rechazo de armónicas. En la medida que el grado de Taylor K aumenta, los $O$-splines tienden a la de un filtro pasabajas ideal de forma perfectamente rectangular. Note que estos filtros ofrecen una nueva secuencia de aproximación al filtro ideal, similar a los de Butterworth o del Coseno Levantado. Aunque en el caso de los filtros DTFT, éstos vengan con sus propios diferenciadores, como se verá en la siguiente subsección.

\section{Diferenciadores Pasabanda}

La ventaja de los filtros DTFT es que vienen acompañados con las derivadas de los $O$-splines. La gráfica superior de la figura 2 muestra la primera derivada de los splines de la figura 1. Sus respuestas en frecuencia se grafican abajo. Note que si $H_{k}^{(0)}(f)$ es la respuesta en frecuencia del $k$-ésimo filtro pasabajas, entonces la respuesta en frecuencia del correspondiente diferenciador es $H_{k}^{(1)}(f)=(j 2 \pi f)^{1} H_{k}^{(0)}(f)$ y sus respuestas en frecuencia tienen una ganancia lineal en la banda de paso.

Similarmente, la segunda derivada de los $O$-splines se ilustra en la gráfica superior de la figura 3. De nuevo, estas corresponden a la derivada de los anteriores. Su respuesta en frecuencia está graficada abajo. Estos tienen una ganancia parabólica en la banda de paso ya que $H_{k}^{(2)}(f)=(j 2 \pi f)^{2} H_{k}^{(0)}(f)$.

Finalmente, el renglón de graficas de la figura 4 ilustra los $O$-splines de $K=0$ a $K=3$, y los vectores bajo cada una de ellas, ilustran sus progresivas derivadas de arriba hacia abajo. Como puede verse, los altos grados requieren ventanas de observación más grandes, pero ofrecen más detalles de los eventos dinámicos, y 
mejoran la exactitud paramétrica. Note cómo las diagonales son combinaciones lineales de traslaciones del primer spline de la correspondiente diagonal, indicando que residen en el subespacio que aloja dicho spline. En la siguiente sección, se comparan los sincrofasores y los parámetros estimados con el conjunto de diferenciadores de primer y tercer grado $(K=1$ y $K=3)$.
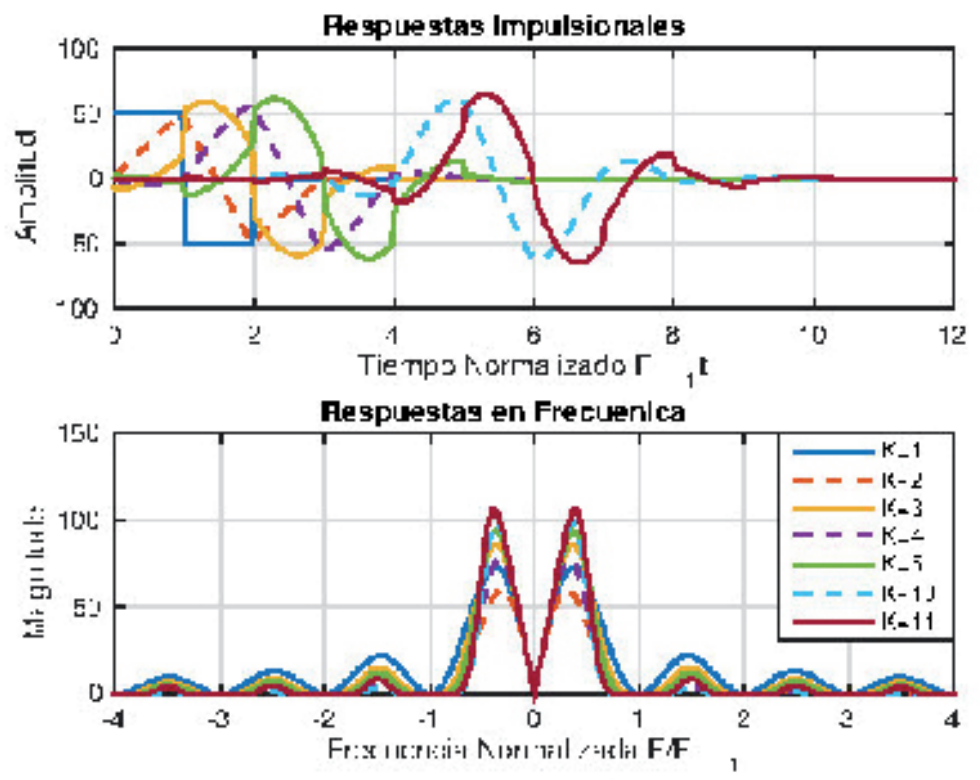

Fig. 2. Primera derivada de las $O$-splines (gráfica superior) y respuesta en frecuencia (gráfica inferior) del primer diferenciador pasabajas, $K=0,1, \ldots, 5$, y 11 .
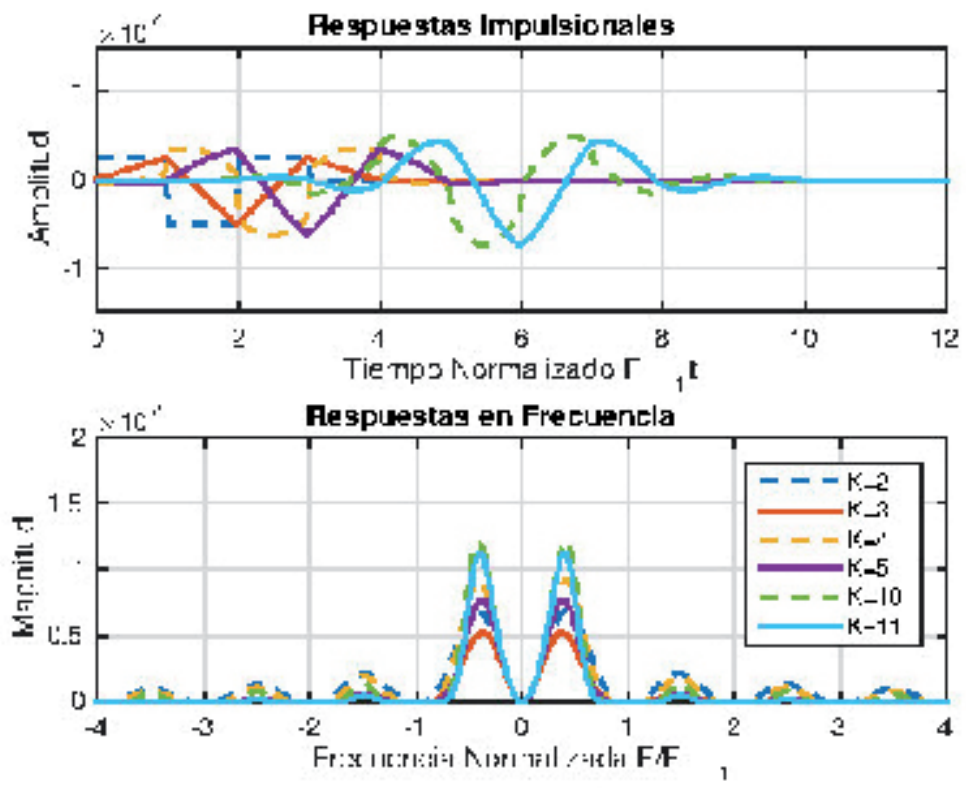

Fig. 3. Segunda derivada de los 0 -splines y respuestas en frecuencia del segundo diferenciador pasabajas, $\mathrm{K}=0,1, \ldots, 5, \mathrm{y} 11$. 

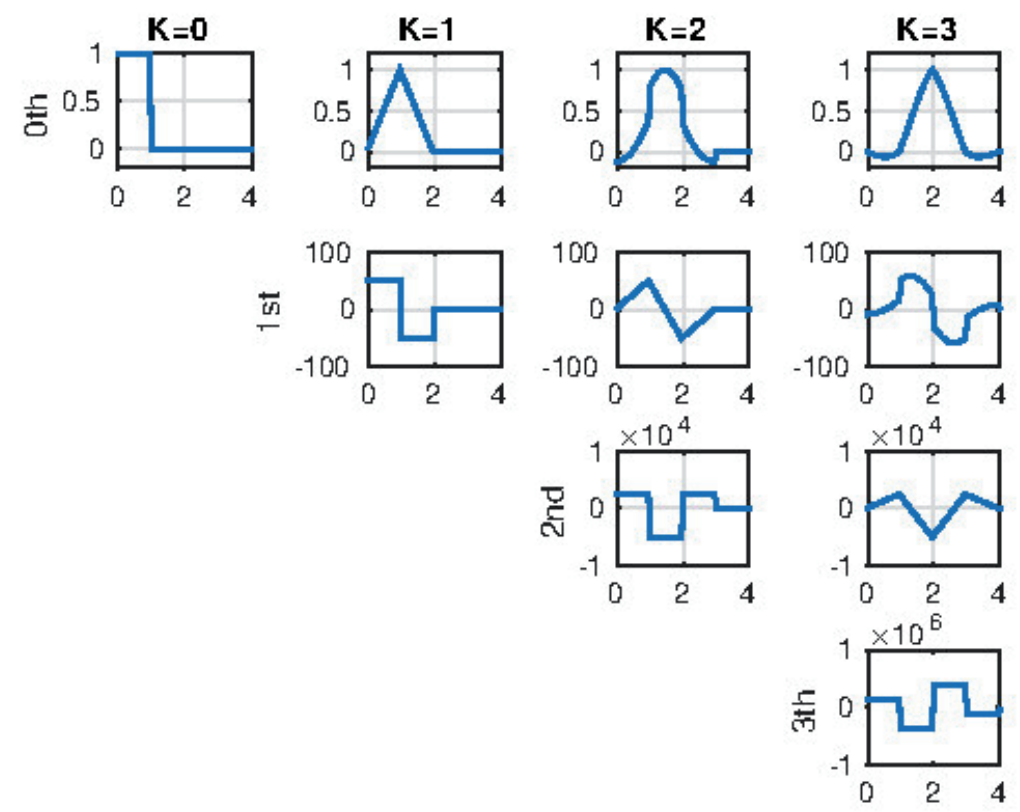

Fig. 4. O-splines (primer renglón) y derivadas de los bancos de filtro DTFT pasabajas (columnas), $K=0,1,2,3$.

\section{RESULTADOS: ESTIMACIÓN DE SINCROFASORES}

La estimación de sincrofasores es una aplicación interesante de la DTFT. Con la DTFT es posible estimar no sólo las excursiones de amplitud y fase de voltajes y corrientes durante una oscilación de potencia, sino también sus fluctuaciones de frecuencia, y su tasa de cambio (ROCOF). En esta sección se ilustran los estimados de voltajes trifásicos de una interesante oscilación real de un sistema de potencia de $50 \mathrm{~Hz}$, cuyas señales fueron grabadas a 20 muestras por período fundamental $(1 \mathrm{kHz})$.

La figura 5 muestra en línea punteada la señal del Voltaje $C$ durante una rápida oscilación, junto con la envolventes de amplitud obtenidas con $\mathrm{K}=1 \mathrm{y}$ $\mathrm{K}=3$, respectivamente. Las estimaciones son calculadas deslizando la ventana de observación punto por punto, produciendo estimados puntuales. Por otra parte, la figura 6 muestra las estimaciones de fase de los tres voltajes. Como puede verse, el caso de oscilación corresponde a un vaivén muy rápido.

La derivada del ángulo de fase ofrece la fluctuación de frecuencia durante la oscilación. La gráfica de arriba en la figura 7 muestra las estimaciones de frecuencia del Voltaje C. Los cruces por la línea $\mathrm{f}=50 \mathrm{~Hz}$ marcan los puntos de máximos y mínimos de la gráfica de fase en la figura 6 . Es evidente que el análisis DTFT puede rastrear muy rápidas excursiones de frecuencia (mucho más grandes que las cotas del estándar). Por otra parte, la gráfica de abajo de la figura 7 ilustra la derivada de frecuencia (ROCOF) del evento en Hz/s. Nuevamente, sus cruces por cero marcan los máximos y mínimos de la curva en la gráfica superior.

Finalmente, la figura 8 muestra el TPE definido en (11), calculado sobre intervalos de un ciclo, entre la señal dada y la estimada, sintetizada con estimados instantáneos (punto a punto). Como se esperaba, los errores obtenidos con $\mathrm{K}=1$ son mayores que los de $\mathrm{K}=3$, sin embargo ambas aproximaciones son muy pobres. 


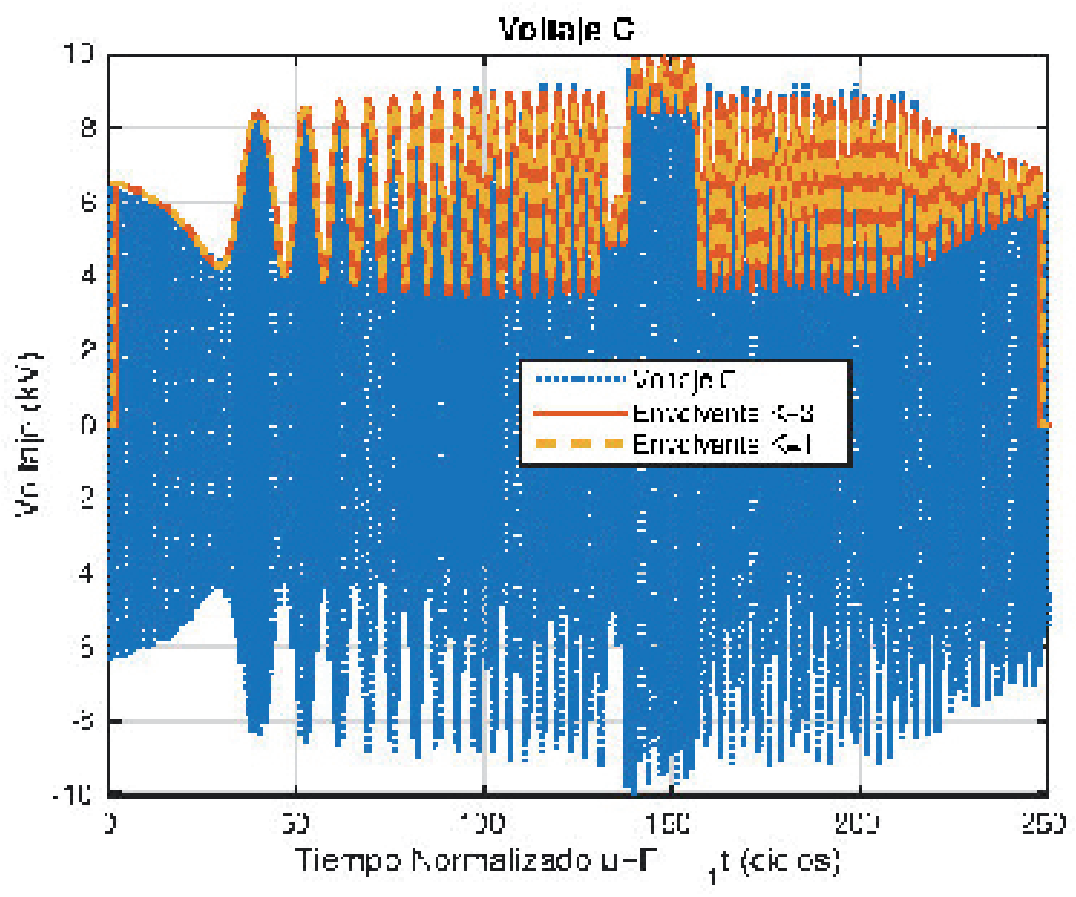

Fig. 5. Voltaje $C$ durante un vaivén de potencia (línea punteada), y envolventes de amplitudes estimadas punto por punto con $\mathrm{K}=1$ (línea a rayas) y K=3 (línea continua).

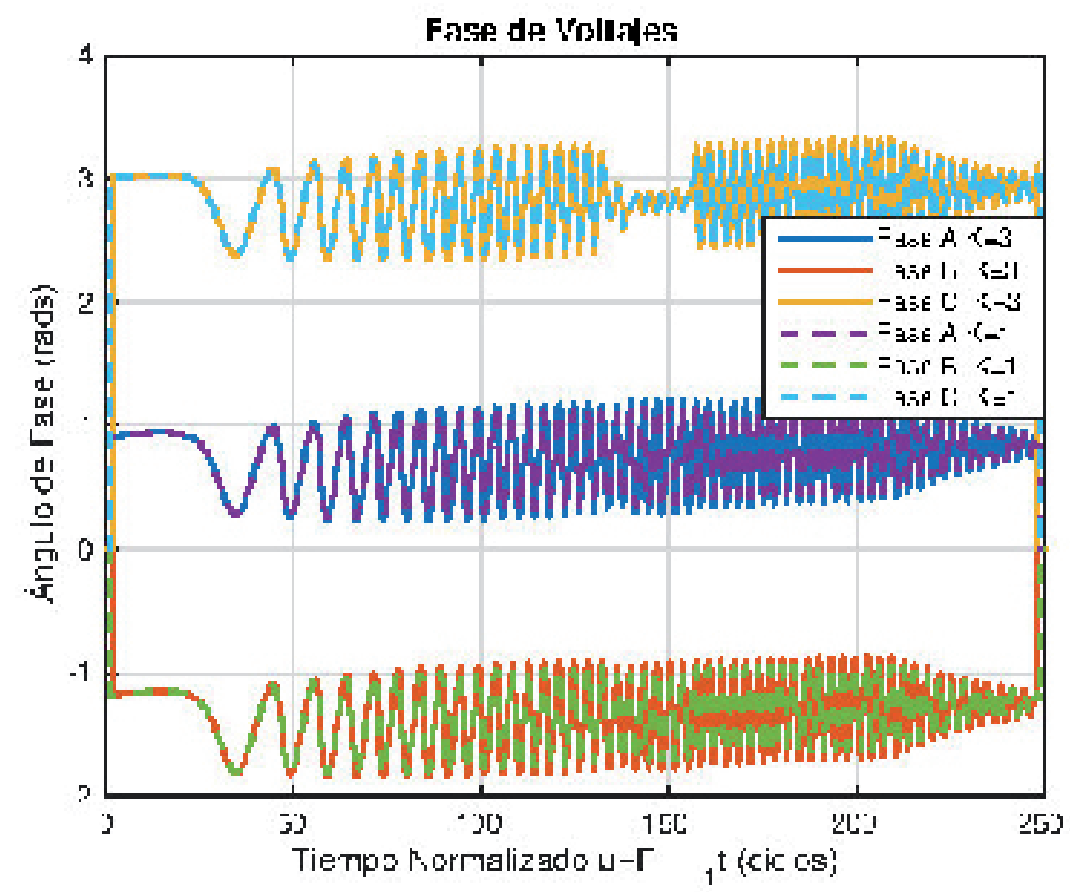

Fig. 6. Ángulo de fase de voltajes A, B, y C, durante la oscilación de potencia calculados con $\mathrm{K}=1$ (línea a rayas) y $\mathrm{K}=3$ (línea continua). 

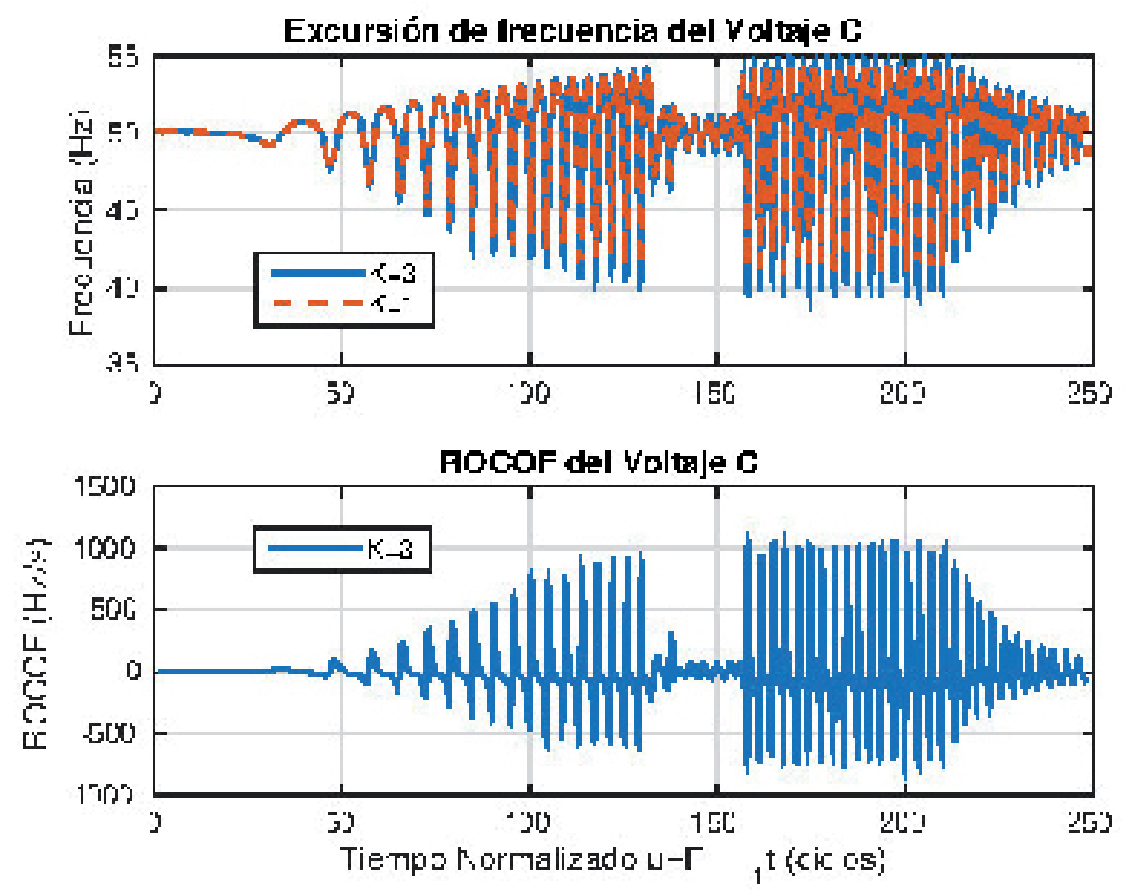

Fig. 7. Excursión de frecuencia del Voltaje $C$ (arriba), con $\mathrm{K}=1$ en línea a rayas y $\mathrm{K}=3$ en línea continua. Al fondo su derivada (ROCOF) estimada con $\mathrm{K}=3$.

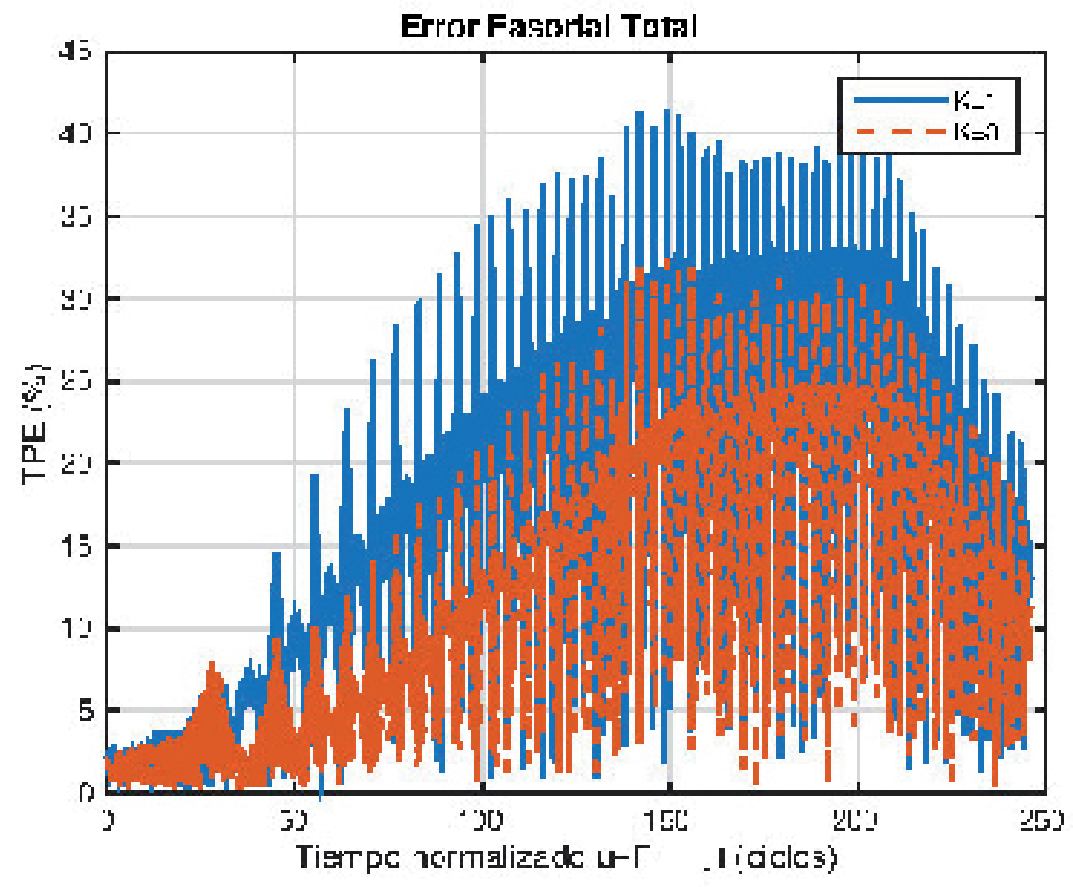

Figure 8: TPEs calculado punto a punto en intervalos de un ciclo, con $\mathrm{K}=1$ y $\mathrm{K}=3$. 


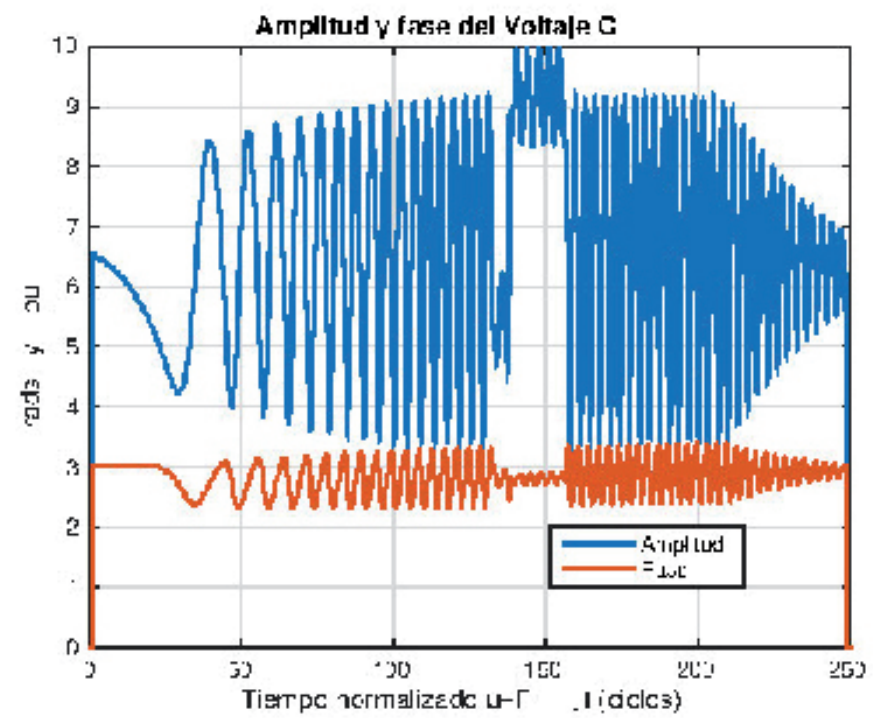

Fig. 9. Estimados de amplitud y fase del Voltaje $\mathrm{C}$ con $\mathrm{O}$-splines de 3 er orden contraídos a dos ciclos.

Aparentemente hemos encontrado una señal anómala para la DTFT. Note los errores pico de la figura 8 se deben a las caídas de frecuencia de la figura 7, y que las mejores aproximaciones se encuentran bajo los topes de las fluctuaciones cóncavas. Además, las formas parabólicas de frecuencia se van haciendo cada vez más angostas cuando el tiempo avanza, desde 10 hasta 3 ciclos. Como los $O$-splines son de 4 períodos, la suposición de fluctuaciones suaves es únicamente válida cuando las fluctuaciones parabólicas son más anchas de 4 ciclos. Esto explica el incremento del error en la figura 8 , hasta los más grandes obtenidos después del 150 avo ciclo.

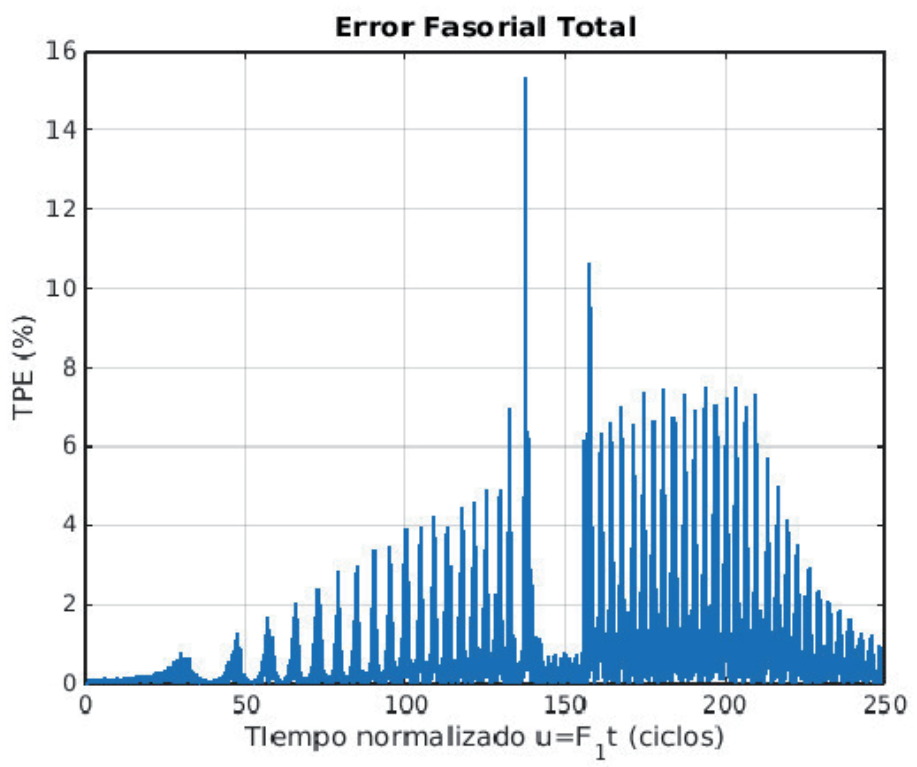

Fig. 10. TPE en Voltaje C obtenido con 0-splines de 3 er orden contraídos a 2-cycles 
Afortunadamente, la DTFT ofrece una amplia gama de soluciones. E1 inconveniente anterior se resuelve submuestreando las curvas flexibles $\mathrm{O}$ de orden 3 por un factor de 2 , y escalándolas para obtener ganancia unitaria en el filtro pasabajas. De esta manera, los $O$-splines son mucho más angostas (de 2 ciclos). La figura 9 muestra las amplitudes y fases estimadas del voltaje $\mathrm{C}$ con estos splines contraídos. Este cambio reduce el TPE como se ilustra en la figura 10. Note que las fluctuaciones parabólicas continúan manifestándose en esta gráfica.

\section{RESULTADOS: SEPARACIÓN DE MODOS DE OSCILACIÓN}

En esta sección se usa la DTFT de 3er orden para separar los modos electromecánicos ${ }^{16}$ de la oscilación de potencia del anterior vaivén. Como las frecuencias de los modos tienen que conocerse a priori, esta aplicación es aún un análisis post mortem. Esta aplicación muestra que la DTFT is capaz de detectar modos con frecuencia modulada.

La oscilación de potencia instantánea aparece en la gráfica superior de la figura 11, y su espectro normalizado se ilustra en la de en medio. Contiene un modo en $f_{0}=0 \mathrm{~Hz}$, y otro en $f_{1}=15.7 \mathrm{~Hz}$. La oscilación tiene también tres pequeños modos perceptibles de alta frecuencia. Una a $f_{2}=100.48 \mathrm{~Hz}$, la otra a $f_{3}=113.04 \mathrm{~Hz}$, y la tercera a $f_{4}=128.74 \mathrm{~Hz}$. Como puede verse, las frecuencias de estos modos no son múltiples de la primera (en $15.7 \mathrm{~Hz}$ ). La gráfica inferior ilustra la respuesta en magnitud de los filtros DTFT empleados en el análisis. Estos fueron diseñados como sigue: Un ciclo fundamental de la oscilación de potencia $(64 \mathrm{~ms})$ contiene 64 muestras. Por tanto, la DTFT debe incluir 32 armónicas. La ventana de observación de los filtros es por tanto de $256 \mathrm{~ms}$. Los tres filtros pasabanda de alta frecuencia se sintonizan en las frecuencias centrales de cada modo.

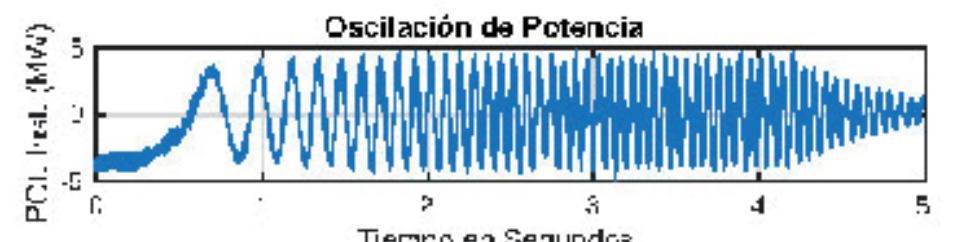

Tiempo en Segundos
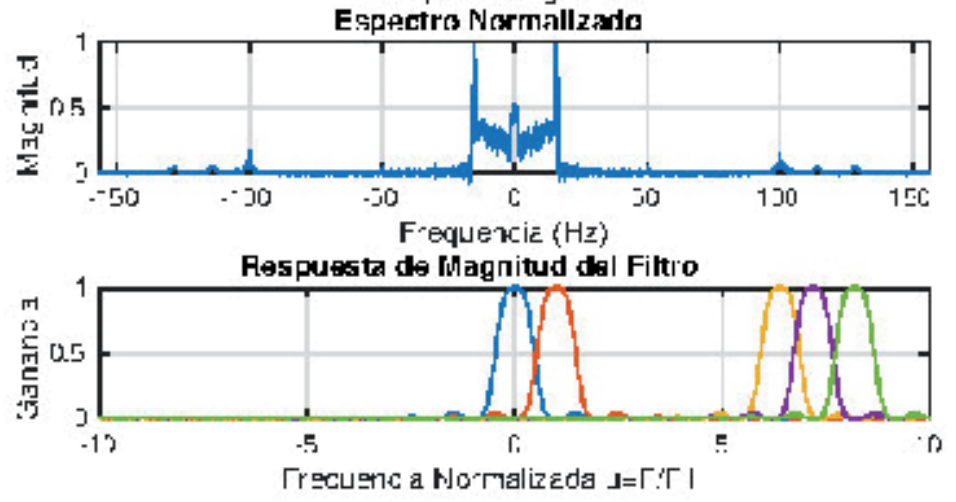

Fig. 11. Oscilación de potencia instantánea (arriba), su espectro normalizado (en medio), y la respuesta en magnitud de los filtros DTFT (al fondo). 
La figura 12 ilustra los modos estimados, y al fondo la oscilación de potencia reconstruida comparada con la original. La secuencia de cada modo $m$ es calculada de los coeficientes instantáneos Taylor Fourier $\xi_{m}(n)$ simplemente como $s_{m}(n)=2\left|\xi_{m}(n)\right| \cos \left(\zeta_{m}(n)\right)$, ya que la contribución de las derivadas $\xi_{m}^{(k>0)}(n)$ en el centro del intervalo es cero. Como puede verse, el modo cero dura los primeros 2 segundos, y el primer modo durante los últimos 4 segundos, mientras que el resto de los modos vive únicamente medio segundo justo después de la mitad del intervalo de observación. Estas gráficas muestran que la DTFT es capaz de separar eventos en tiempo y frecuencia. Divide el evento en tres intervalos: uno antes de la falla, el otro durante la falla, y el último después de la falla. En la gráfica inferior, es patente que la oscilación de potencia reconstruida (línea punteada) se ajusta bien a la oscilación original. La energía del error es $5 \%$ de la energía de la señal de oscilación. Hay un pequeño error debido a que la separación de los dos primeros modos no es perfecta, es decir que ellos comparten energía en las bandas de transición de los filtros correspondientes (ver gráfica inferior de la figura 11).
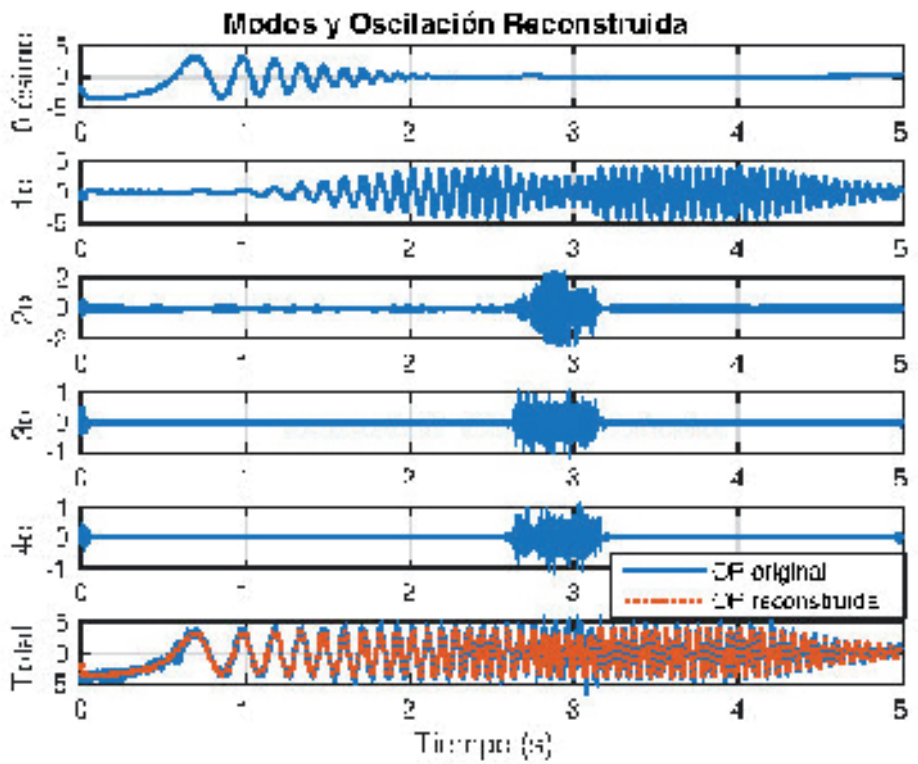

Fig. 12. Modos de oscilación y al fondo la oscilación de potencia sintética, comparada con la señal original de oscilación.

Las técnicas de descomposición de modos tradicionales generalmente consideran la componente cero como una constante que se elimina mediante la sustracción del promedio de la señal antes del análisis. Esta consideración es una herencia de la manera fourierana de pensar. La descomposición de modos con la DTFT muestra claramente que, en general, el modo cero fluctúa, y debe considerarse como un modo de oscilación per se, ya que contiene información dinámica importante, como se muestra en lo que sigue.

La amplitud del modo cero y su primera derivada se muestra en las gráficas de la figura 13. Note que los cruces por cero de la primera derivada corresponden a los máximos y mínimos de la amplitud. Lo mismo es válido para la segunda derivada mutatis mutandis. Las derivadas de la DTFT son muy exactas. 

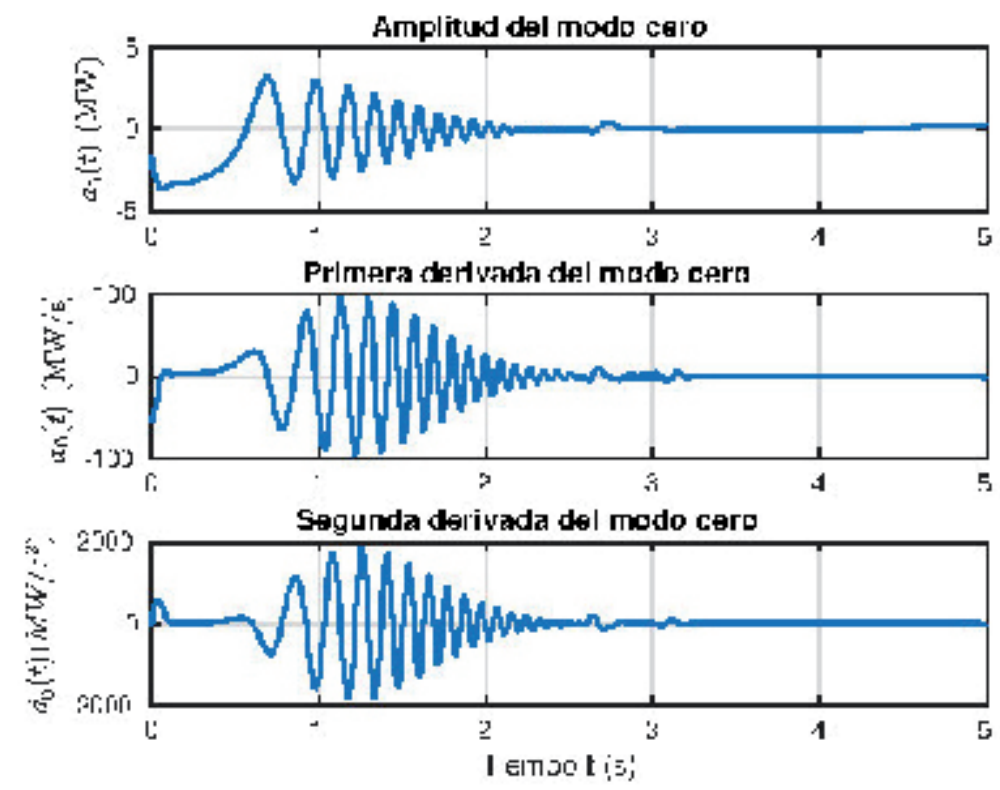

Fig. 13. Amplitud del modo cero, y sus primeras dos derivadas.

Por otra parte, la figura 14 muestra en la gráfica superior la envolvente de amplitud del primer modo en MW, y al fondo su primera derivada en MW/s. Hay una patente infiltración del modo cero, que es amplificada por el diferenciador. Cuando este modo desaparece en el intervalo postfalla, se suavizan tanto la amplitud como su derivada. Note que dos picos delimitan la duración de la falla.
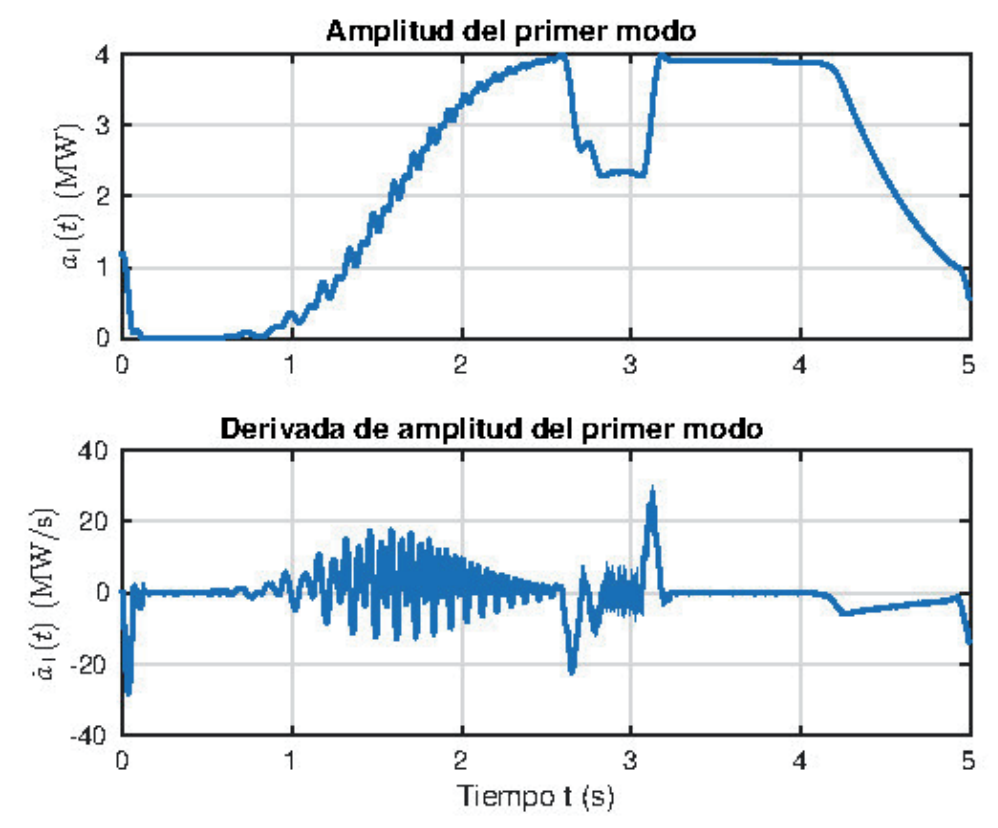

Fig. 14. Envolvente de amplitud del primer modo en MW (gráfica superior), y su primera derivada en $\mathrm{MW} / \mathrm{s}$ (gráfica inferior). 
Por último, la gráfica superior de la figura 15 muestra la fase y la del fondo su derivada. De nuevo, la infiltración del modo cero es patente en la gráfica de abajo. Sin embargo, es evidente que el primer modo es de frecuencia modulada, ya que su frecuencia inicia en $0 \mathrm{~Hz}$, y crece exponencialmente con pequeñas fluctuaciones hasta alcanzar $15.6 \mathrm{~Hz}$ en $\mathrm{t}=3 \mathrm{~s}$, donde se mantiene hasta el final del registro. Esta evolución de la frecuencia se muestra con menos resolución en el espectrograma ilustrado en la Fig. 4, calculado con una secuencia de transformadas rápidas de Fourier (FFTs) usando una ventana rectangular de 4 ciclos (256 muestras). La variación de frecuencia es también patente en la oscilación de potencia misma en la gráfica superior de la figura 16, en la cual los intervalos pico a pico de la oscilación disminuyen paulatinamente en la medida que la frecuencia aumenta. Por lo tanto, la DTFT descubre una frecuencia dinámica no percibida por los métodos tradicionales de descomposición de modos, los cuáles asumen modos de frecuencia constante.
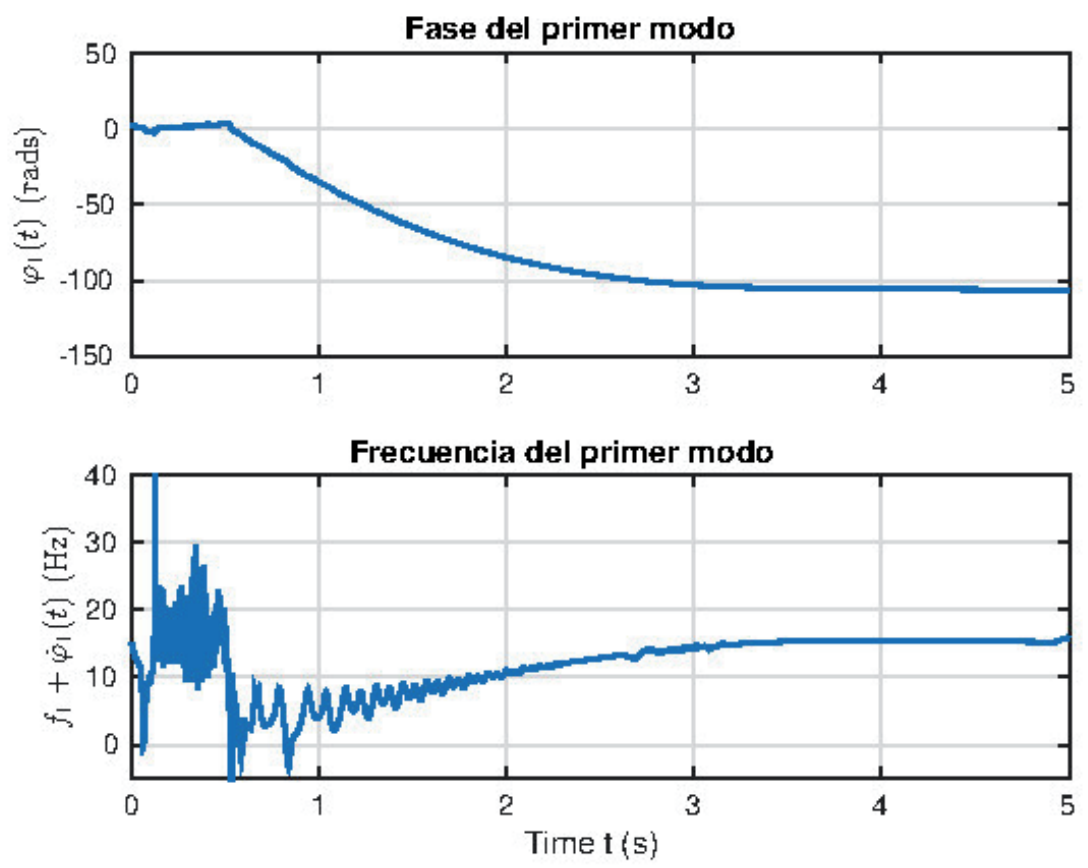

Fig. 15. Fase del primer modo (gráfica superior) y su frecuencia (gráfica inferior).

Los mismos estimados pueden obtenerse para los últimos tres modos en la falla. Pero los analizados son suficientes para mostrar que la DTFT ofrece separación tiempo frecuencia, y puede tratar con modos de frecuencia modulada. En ${ }^{16} \mathrm{el}$ rendimiento del análisis con la 3 er DTFT se comparó con los métodos de Prony, ${ }^{11} \mathrm{el}$ algoritmo de realización eigensistema (ERA), ${ }^{25}$ y el Matrix Pencil. ${ }^{26}$. De los modos interárea analizados en ${ }^{16}$ (en $0.133,0.399,0.532,0.665$, y $\left.0.798 \mathrm{~Hz}\right)$, se concluyó que la DTFT puede aplicarse a oscilaciones de baja frecuencia. Sin embargo, note que el análisis de modos de más baja frecuencia requieren ventanas más largas; por ejemplo, un modo de $0.1 \mathrm{~Hz}$ necesita una ventana de $40 \mathrm{~s}$. Sin embargo, dichos modos pueden también extraerse con el filtro pasabajas. 


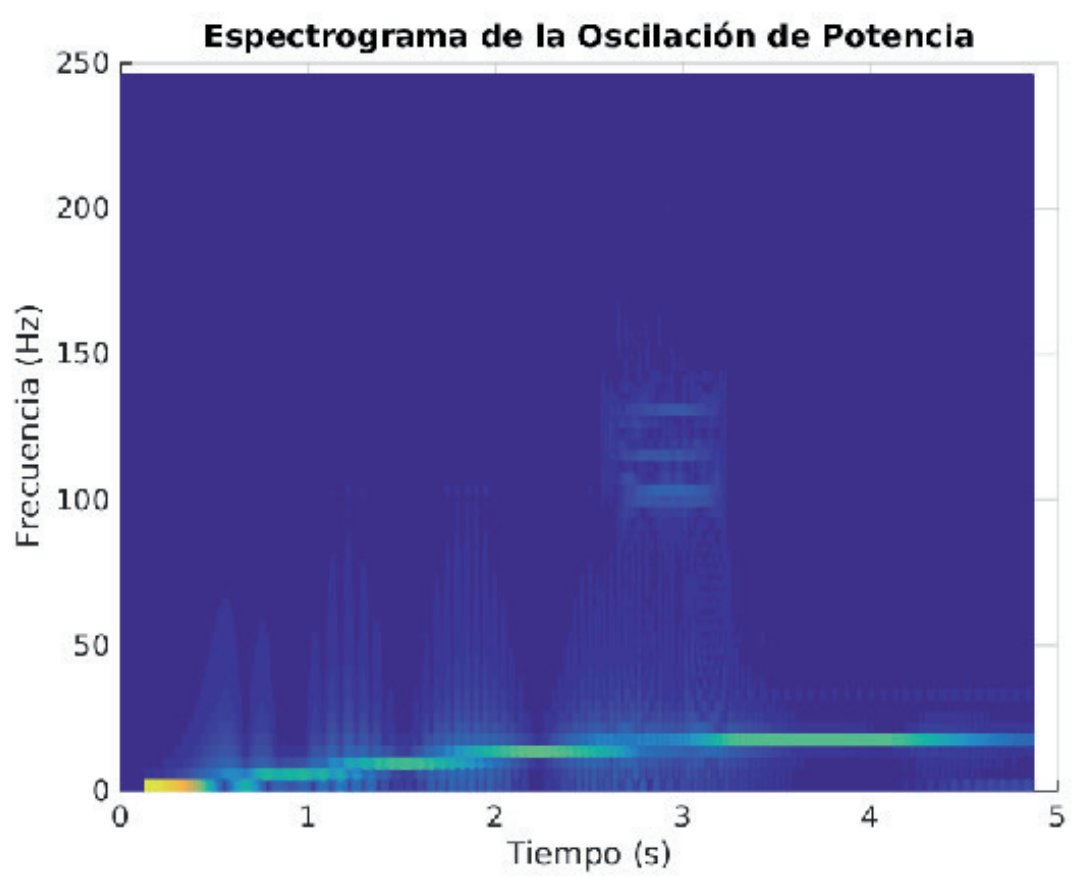

Fig. 16.: Espectrograma de la oscilación de potencia.

\section{DISCUSIÓN}

Con respecto a la aplicación de sincrofasores, los $O$-splines ofrecen un serie de elegantes soluciones para las clases P y M del Estándar. ${ }^{4}$. Los resultados muestran que los estimados de la DTFT ofrecen información dinámica mucho más rica que la especificada por dicho Estándar. Además, el error fasorial total evalúa la calidad del conjunto de parámetros estimados. Con relación al problema de detección de modos de oscilación, la DTFT ofrece separación tiempo frecuencia de modos modulados en frecuencia o modos de frecuencia constante. Finalmente, la elegante teoría matemática detrás de la DTFT ofrece una solución unificante de dos problemas importantes en medición de sistemas de potencia de área amplia, tradicionalmente considerados como completamente independientes. Sin embargo, cabe señalar que la DTFT asume que la energía espectral de la señal se concentra alrededor de ciertas frecuencias, y que en la ventana temporal de observación la envolvente compleja sea tan suave como para ser aproximada por un polinomio de Taylor. En consecuencia, la DTFT es incapaz de extraer transitorios muy rápidos con un espectro demasiado amplio, ya que la separaría en varias componentes.

\section{CONCLUSIÓN}

Los $O$-splines permiten sintonizar filtros DTFT en un cierto número de frecuencias, reduciendo la complejidad numérica de la DTFT. Los coeficientes Taylor Fourier son muy útiles para el análisis multiresolución de señales oscilatorias de frecuencia fluctuante. El caso ilustrado de señal demuestra que existen señales de sistemas de potencia reales con fluctuaciones mucho más complejas que las señales de prueba del estándar de sincrofasores del IEEE. En lo concerniente al problema de separación de modos, la DTFT es capaz de separarlos 
en tiempo y en frecuencia, incluyendo el modo oscilatorio cero, tradicionalmente considerado como constante.

La DTFT ofrece una única teoría para resolver dos problemas importantes de sistemas de potencia considerados tradicionalmente como independientes, y abre el camino a interpretaciones más precisas y exactas de los eventos oscilatorios, dando lugar a nuevo conocimiento. La siguiente etapa es usar los $O$-splines con frecuencia adaptiva, y obtener soluciones en tiempo real para la separación de modos de oscilación.

\section{AGRADECIMIENTO}

El autor agradece amablemente a Jörg Blumschein por haber aportado el registro de las señales de oscilación analizadas en éste artículo.

\section{REFERENCIAS}

1. J. G. Proakis and D. K. Manolakis, Digital Signal Processing, 4th ed. Upper Saddle River, N.J: Pearson, 2006.

2. M. A. Platas-Garza and J. A. De la O Serna, "Dynamic harmonic analysis through Taylor-Fourier transform," IEEE Trans. Instrum. Meas., vol. 60, no. 3, pp. 804-813, 2011. [Online]. Available: http://dx.doi.org/10.1109/ TIM.2010.2064690

3. J. A. de la O Serna, "Analyzing power oscillating signals with the o-splines of the discrete taylor-fourier transform," IEEE Trans. Power Syst., vol. 33, no. 6, pp. 7087-7095, Nov 2018.

4. "IEEE standard for synchrophasor measurements for power systems," IEEE Std C37.118.1-2011 (Revision of IEEE Std C37.118-2005), pp. 1-61, Dec 2011.

5. A. G. Phadke and B. Kasztenny, "Synchronized phasor and frequency measurement under transient conditions," IEEE Trans. Power Del., vol. 24, no. 1, pp. 89-95, Jan 2009.

6. R. Ghiga, K. Martin, Q. Wu, and A. Nielsen, "Phasor measurement unit test under interference conditions," IEEE Trans. Power Del., vol. PP, no. 99, pp. 1-1, 2017, DOI:10.1109/TPWRD.2017.2691356.

7. D. Macii, D. Petri, and A. Zorat, "Accuracy analysis and enhancement of DFT-based synchrophasor estimators in off-nominal conditions," IEEE Trans. Instrum. Meas., vol. 61, no. 10, pp. 2653-2664, Oct 2012.

8. M. Karimi-Ghartemani, B. T. Ooi, and A. Bakhshai, "Application of enhanced phase-locked loop system to the computation of synchrophasors," IEEE Trans. Power Del., vol. 26, no. 1, pp. 22-32, Jan 2011.

9. I. Kamwa, A. K. Pradhan, and G. Joos, "Adaptive phasor and frequencytracking schemes for wide-area protection and control," IEEE Trans. Power Del., vol. 26, no. 2, pp. 744-753, April 2011.

10.J. S. Gasca and D. Trudnowski, "Identification of electromechanical modes in power systems," IEEE Power and Energy Society, Task Force on Identificatioin of electromechanical modes, Tech. Rep., June 2012. 
11. J. F. Hauer, C. J. Demeure, and L. L. Scharf, "Initial results in Prony analysis of power system response signals," IEEE Trans. Power Syst., vol. 5, no. 1, pp. 80-89, Feb 1990.

12.J. Rommes, N. Martins, and F. Freitas, "Computing rightmost eigenvalues for small-signal stability assessment of large scale power systems," in IEEE PES General Meeting, July 2010, pp. 1-1.

13.J. W. Pierre, D. J. Trudnowski, and M. K. Donnelly, "Initial results in electromechanical mode identification from ambient data," IEEE Trans. Power Syst., vol. 12, no. 3, pp. 1245-1251, Aug 1997.

14.A. R. Messina and V. Vittal, "Nonlinear, non-stationary analysis of interarea oscillations via Hilbert spectral analysis," IEEE Trans. Power Syst., vol. 21, no. 3, pp. 1234-1241, Aug 2006.

15.A. Q. Zhang, L. L. Zhang, M. S. Li, and Q. H. Wu, "Identification of dominant low frequency oscillation modes based on blind source separation," IEEE Trans. Power Syst., vol. PP, no. 99, pp. 1-1, 2017.

16.J. A. De la O Serna, J. M. Ramirez, A. Zamora Mendez, and M. R. A. Paternina, "Identification of electromechanical modes based on the digital Taylor-Fourier Transform,” IEEE Trans. Power Syst., vol. 31, no. 1, pp. 206-215, 2016. [Online]. Available: http://dx.doi.org/10.1109/TPWRS.2015.2403290

17. J. A. De la O Serna, "Synchrophasor measurement with polynomial phaselocked-loop Taylor-Fourier filters," IEEE Trans. Instrum. Meas., vol. 64, no. 2, pp. 328-337, 2015. [Online]. Available: http://dx.doi.org/10.1109/ TIM.2014.2344333

18.I. Daubechies, Ten Lectures on Wavelets. Society for Industrial and Applied Mathematics, 1992. [Online]. Available: http://epubs.siam.org/doi/abs/10.1 137/1.9781611970104

19.J. A. De la O Serna, "Dynamic phasor estimates for power system oscillations," IEEE Trans. Instrum. Meas., vol. 56, no. 5, pp. 1648-1657, 2007. [Online]. Available: http://dx.doi.org/10.1109/TIM.2007.904546

20.M. A. Platas-Garza and J. A. De la O Serna, "Dynamic phasor and frequency estimates through maximally flat differentiators," IEEE Trans. Instrum. Meas., vol. 59, no. 7, pp. 1803-1811, 2010. [Online]. Available: http://dx.doi. org/10.1109/TIM.2009.2030921

21.M. Vetterli, K. Kovacevic, and V. K. Goyal, Foundations of Signal Processing, 3rd ed. Cambridge, UK: Cambridge University Press, 2014, available at http:// www.fourierandwavelets.org/. 